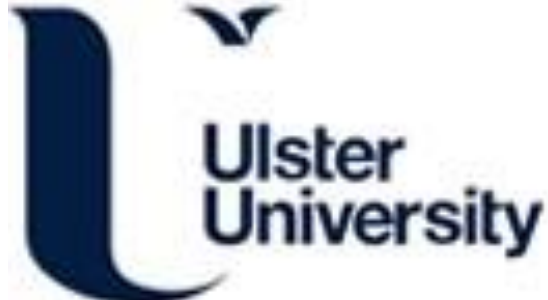

\section{uMoDT: an unobtrusive multi-occupant detection and tracking using robust Kalman filter for real-time activity recognition}

Razzaq, M. A., Quero, J. M., Cleland, I., Nugent, CD., Akhtar, U., Bilal, H. S. M., Ur Rehman, U., \& Lee, S. (2020). uMoDT: an unobtrusive multi-occupant detection and tracking using robust Kalman filter for real-time activity recognition. Multimedia Systems, 26(5), 553-569. https://doi.org/10.1007/s00530-020-00664-7

Link to publication record in Ulster University Research Portal

\section{Published in:}

Multimedia Systems

Publication Status:

Published (in print/issue): 31/10/2020

DOI:

10.1007/s00530-020-00664-7

\section{Document Version}

Author Accepted version

\section{General rights}

Copyright for the publications made accessible via Ulster University's Research Portal is retained by the author(s) and / or other copyright owners and it is a condition of accessing these publications that users recognise and abide by the legal requirements associated with these rights.

\section{Take down policy}

The Research Portal is Ulster University's institutional repository that provides access to Ulster's research outputs. Every effort has been made to ensure that content in the Research Portal does not infringe any person's rights, or applicable UK laws. If you discover content in the Research Portal that you believe breaches copyright or violates any law, please contact pure-support@ulster.ac.uk. 


\title{
uMoDT: An unobtrusive Multi-occupant Detection and Tracking using robust Kalman filter for real-time activity recognition
}

\author{
Muhammad Asif Razzaq1(D, Javier Medina Quero'(D), Ian Cleland ${ }^{3}$ (D), \\ Chris Nugent $^{3}$ (D), Usman Akhtar ${ }^{1}$, Hafiz Syed Bilal Ali ${ }^{1}$, Ubaid Ur \\ Rehman $^{1}$, Sungyoung Lee ${ }^{1}$
}

Received: date / Accepted: date

\begin{abstract}
Human activity recognition (HAR) is an important branch of human-centered research. Advances in wearable and unobtrusive technologies offer many opportunities for HAR. While much progress has been made in HAR using wearable technology, it still remains a challenging task using unobtrusive (non-wearable) sensors. This paper investigates detection and tracking of multi-occupant HAR in a smart-home environment, using a novel low-resolution Thermal Vision Sensor (TVS). Specifically, the research presents the development and implementation of a two-step framework, consisting of a Computer Vision (CV) based method to detect and track multiple occupants combined with Convolutional Neural Network (CNN) based HAR. The proposed algorithm uses frame-difference over consecutive frames for occupant detection, a set of morphological operations to refine identified objects, and features are extracted before applying a Kalman filter for tracking. Laterally, a 19-layer CNN architecture is used for HAR and afterward the results from both methods are fused using time interval based sliding window. This approach is evaluated through a series of experiments based on benchmark Thermal Infrared datasets (VOTTIR2016) and multi-occupant data collected from TVS. Results demonstrate that the proposed framework is capable of detecting and tracking $88.46 \%$ of multi-
\end{abstract}

\footnotetext{
1 Ubiquitous Computing Lab, Department of Computer Engineering, Kyung Hee University, 1 Seocheon-dong, Giheunggu, Yongin-si, Gyeonggi-do 446-701, Korea; (asif.razzaq, usman, bilalrizvi, ubaid.rehman, sylee)@oslab.khu.ac.kr

2 Department of Computer Science, University of Jaén, Campus Las Lagunillas, Jaén 23071, Spain; jmquero@ujaen.es

3 School of Computing and Mathematics, Ulster University, Jordanstown, BT37 0QB, Northern Ireland, UK; (cd.nugent, i.cleland)@ulster.ac.uk
}

occupants with a classification accuracy of $90.99 \%$ for HAR.

Keywords Human activity recognition - Image processing $\cdot$ Object detection $\cdot$ Tracking $\cdot$ Classification

\section{Introduction}

Over several decades, advances in pervasive computing has offered great promise towards the potential of indoor localization and Human Activity Recognition (HAR) 11. Over this period, significant research effort has been targeted towards the creation of solutions that can reliably monitor individuals through the use of onbody wearable sensors, dense sensors, and vision sensors 2. Whilst results utilizing on-body sensors has improved greatly, wearable solutions are often said to be impractical, as they can be difficult to carry or inconvenient to wear continuously [3]. Additionally, vision sensors capable of capturing RGB or grayscale images have been studied intensively within the Computer Vision $(\mathrm{CV})$ domain. The use of cameras, however, raises serious privacy concerns 4 .

Recently, researchers have been investigating the potential of deploying unobtrusive, inexpensive and low resolution Thermal Vision Sensors (TVS) for occupant detection and pervasive sensing [5]. Similar to traditional vision based approaches, TVS suffer from same limitations for handling complex object appearances due to shape deformation, low resolution, varying number of objects, pose variation, motion estimation, and object re-identification [6. TVS do, however, address some of the challenges as they tend to be more robust to illumination changes, can operate even in complete darkness and offer less intrusion on user's privacy 7 . 
The majority of research into HAR has focused on single occupant environments. Nevertheless, living environments are usually inhabited by more than one person. Therefore, HAR in the context of multi-occupancy would provide a more practical solution, however, also more challenging. The difficulty with multi-occupant HAR stems from two related challenges in occupant identification, known as data association, and the diversity of human activities.

In $\mathrm{CV}$, object tracking remains one of the most significant research challenges [8, 9]. This becomes even more complex when using TVS for monitoring multi-occupants, as data only corresponds to variation in temperature. Therefore a different strategy is required for identification and re-identification of the occupants [10]. The aforementioned challenges are addressed by proposing and implementing a robust CVbased integrated framework for multi-occupant detection, tracking and HAR based on TVS.

The remainder of the article is organized as follows: Sect. 2 presents a review of related work; Sect. 3 formulates the problem and introduces the proposed framework describing our pragmatic approaches for multioccupant tracking and HAR; Sect. 4 presents experimental details; Sect. 5 presents both quantitative and qualitative evaluations and comparisons on thermal frame sequence and VOT-TIR2016 benchmarks; Sect.6 offers concluding remarks with a discussion about future improvements.

\section{Related work}

Multi-object Tracking (MOT) in CV domain has been studied for decades and has attracted a lot of research attention. It is, however, still far from solved regarding HAR [11]. Many solutions exist for HAR in a controlled environment. These solutions mostly involve the deployment of numerous wearable and pervasive sensors [12, which can lead to increased cost, privacy concerns and more often inconvenience. To alleviate these challenges, attention of the research community has directed to low-cost unobtrusive sensors 13 .

TVS are an excellent candidate for pervasive sensing due to their inexpensive nature, portability, limited maintenance requirement and lower privacy issues compared to traditional cameras. Hevesi et al. [14] have illustrated that such a sensing modality can be deployed for indoor HAR and monitoring of sedentary behavior of a single occupant in an office environment. Solutions based on TVS mostly require CV based approaches for locating moving objects by identifying them as a region of interest (ROI) in a frame sequence. Detection of a ROI is deemed as the first step in most CV- based applications [15. It may involve various techniques such as: (1) thresholding, which yields low accuracy and is of lesser use in current applications [16] (2) multi-resolution processing which faces challenges for detecting objects during congestion [17]; (3) edge detection which has challenges in deriving an ROI where the shape of object is highly dynamic; (4) inter-frame differencing which uses consecutive frames for detecting an ROI but can only be considered for a sequence of a shorter duration [18; (5) an optical flow based detection which requires a large number of frames resulting in poor performance; (6) background subtraction which extract objects not belonging to the background, however, this technique requires a static background as an initialization.

Regarding MOT various techniques [19] have also been proposed by the research community. These techniques focus on addressing common challenges such as frequent occlusions, identical appearances, track management and interaction among objects. No single approach currently exists which can address all of these challenges. MOT in any visual tracking system usually involves three functional models [20]: (1) appearance model, which describes the object and distinguishes it from the non-objects; (2) motion model which characterizes the current and predicts the future states of an object by tracking their trajectories; (3) searching strategy which helps to identify and match an object based on the appearance model in a frame sequence.

Motion models have gained significant attention for object state estimation. They operate by producing accurate motion affinity models in a linear motion space, which can be used to predict object position [21. Thus, it reduces the search space by capturing the dynamic behavior of the object. To solve the linear tracking problem, where continuity of moving objects is not abrupt, Kalman filtering (KF) is often used [22]. This approach can track moving objects using their center of gravity 23. KF is a linear state-space motion model proved to be an optimal tracker suitable for practical applications. It promises a good compromise between computational complexity and performance for object tracking by utilizing a point-based approach in learning statistical features 24. It uses identified features and uncertainty information to estimate different states of an object through the successive frames. KF may, however, experience object drifting due to the loss of an object's appearance information in a frame sequence. The object drifting complexities require efficient object refinement schemes to analyze object motion properties leading to proper data association [25. Yilmaz et al. 21 addressed some of the issues and complexities related to data associations through a joint solution for 
state estimation. Choi et al. 26] formulated the problem of multi-occupancy and resolved it through multiple target tracking. They merged the problems of HAR and tracking into a single probabilistic graphical model for tracking individual actions. Similarly, an adaptive framework was also proposed by Shen et al. 27] to identify the correct state of the targets. They suggested the use of an adaptive detection algorithm for MOT task to refine the detection targets and minimize the detection errors.

In order to classify Activities of Daily Living (ADLs), it has been observed that CNN have shown superior performance over the traditional Machine Learning (ML) approaches such as Support Vector Machines [28] and feed-forward neural networks [29]. The visual object recognition tasks [30. can be performed over the raw low-resolution TVS frames using CNN, which is easier to train by adjusting a few parameters and inter-layer connections. It extracts meaningful features without requiring domain knowledge and with minimum preprocessing over a stacked sequence of frames [31. The CNN model has the capability to extract multiple motion features encoded in the adjacent TVS frames for automatic classification of ADLs [32.

The current work is closely related to 4 in which the authors proposed a system for indoor player tracking captured through the thermal camera at a sports arena and pedestrian tracking in a courtyard. Ray et al. 33. proposed a detection algorithm, which does not depend on any prior background knowledge for object detection and also does not require initialization. Similarly, Leira et al. 34] considered the problem of small unmanned aerial vehicles equipped with thermal cameras for real-time target detection and tracking at sea using the KF based technique. Tiwari et al. [35] highlighted the research gaps for video-based HAR. They suggested designing an approach to improve the robustness of the detection and tracking algorithms by increasing the number of occupants, which can be tracked over a sequence.

The purpose of this study is to propose a framework for moving object detection, tracking and classification of ADLs with increased performance using lowresolution thermal video frames. To achieve this goal, an implementation using a KF was devised by building a robust object appearance model with morphological feature refinements. It also involves the Hungarian algorithm for data association per frame [27. Additionally, this study evaluates the robustness of the integrated framework to detect and track ADLs of the users using low-resolution TVS. For this, the solution was tested using comprehensive experimental analysis drawing quantitative and qualitative comparisons. Ro-

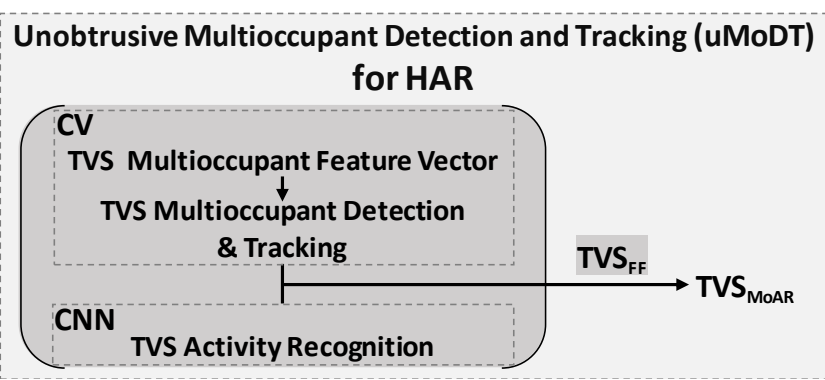

Fig. 1: Overview of proposed solution strategies as uMoDT framework

bust tracking systems, such as [36], mostly involve an appearance and motion model to track the candidate states of the target. Computational complexity, however, increases proportionally with the increase in the number of targets to be tracked [37. Therefore, a joint optimization is essential for MOT. Most MOT research focuses on tracking-by-detection methods, however, an extension to it, by classifying the activities may result in boosting the overall effectiveness of these methods.

\section{Proposed uMoDT framework}

This section initially outlines the design challenges before presenting the algorithmic solutions and then detailing the overall framework.

\subsection{Overview}

The main challenges in CV-based object detection and tracking applications are correct identification of ROI, reliable and efficient handling of moving objects alongwith their inter-frame associations. These challenges, however, become even more complex for interacting multi-objects, which may have erratic movements represented by low-resolution appearances in a frame sequence. For this, an efficient method is required to predict their motion and manage data association 38 . Additionally, recognition of interaction amongst objects and classification of activities is also a computationally intensive task and requires a more robust process. This further requires a trade-off when implementing the above-mentioned methods in a more efficient manner for a complete, coherent and correct detection, tracking and classification of an occupant's activities. To address the aforementioned challenges, as presented in Fig. 1. we propose a unified scalable unobtrusive Multioccupant Detection and Tracking $(u M o D T)$ framework, 


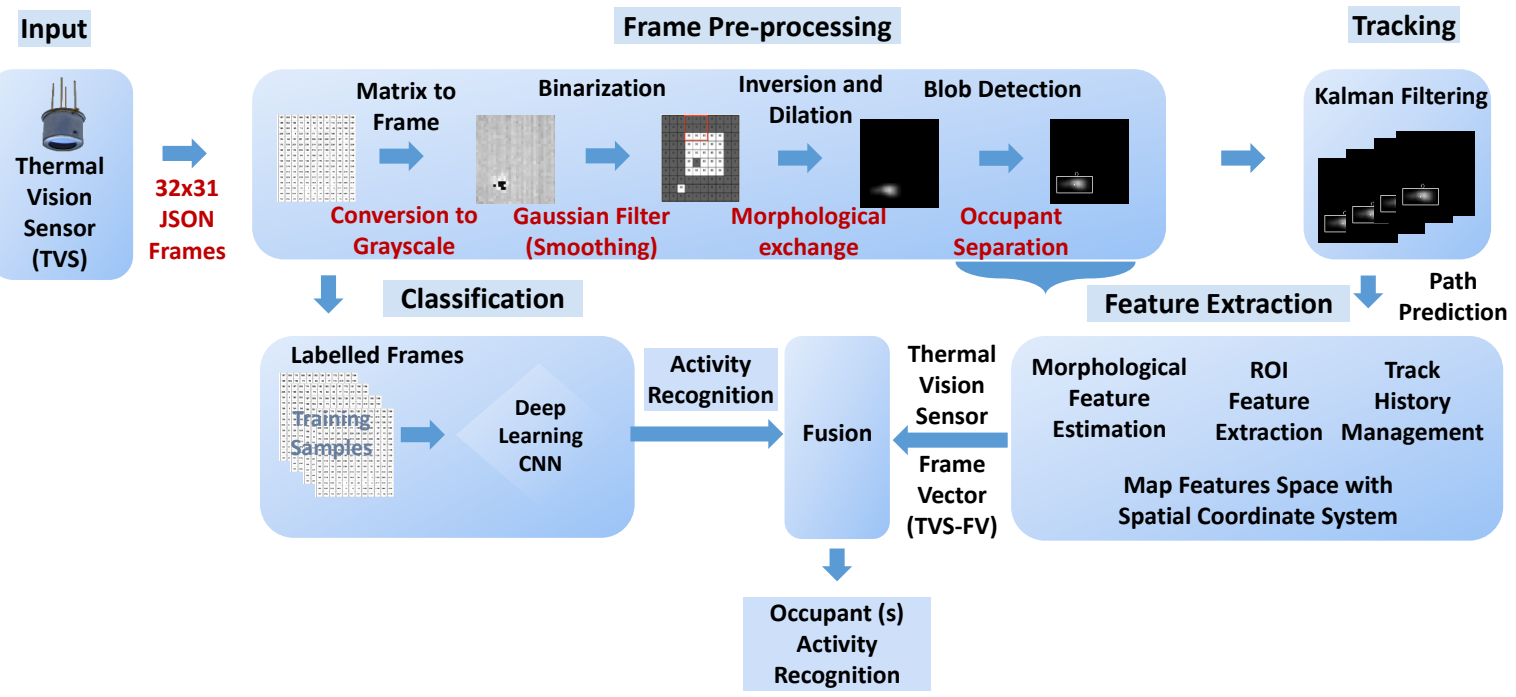

Fig. 2: Proposed unobtrusive Multi-occupant Detection and Tracking (uMoDT) framework for HAR

which detects, tracks and recognizes different indoor activities under multioccupancy using TVS.

The $u M o D T$ framework addresses six strategies as described below:

- We propose an online framework, which uses a CVbased algorithm, with improved morphological features, for an automatic multi-target initialization using frame differencing with an optimum threshold.

- We rely on refined morphological characteristics, which ensure efficient detection and tracking accuracy over the dynamic patterns for nonrigid moving targets per-frame.

- We use the Hungarian method for track assignment problem with an approach for maintaining an association history of re-identified tracks of individual moving objects per-frame.

- The proposed framework is validated using a dataset gathered at Smart Environments Research Group (SERG) laboratory from the Ulster University, UK. It proved to be computationally robust and achieves a promising tracking accuracy in comparison with other MOT methods.

- We also demonstrated quantitative evaluations on the publicly available dataset for the VOT-TIR2016 challenge proving the practicality and efficacy of the proposed framework with the state-of-the-art.

- Additionally, we propose to apply a CNN architecture to extract and learn spatial features from multiple successive Thermal Vision Sensor Frame (TVSF) for individual action recognition.
The focus of the presented work is to simultaneously detect multi-occupants as well as recognize their activities frame-by-frame from TVS. It also requires a solution for resident data association in a smart-home environment, which is accomplished by unifying two different approaches. Firstly, using the CV-based technique, which detects, tracks, and monitors the occupant within the controlled area by observing a robust frame difference between the consecutive frames. Secondly, the CNN layers are invoked by the TVS frame sequence $\left(T V S_{-} F_{s e q}\right)$, which recognizes the occupant's individual activities such as Walking, Standing, Sitting, Fall down. Finally, the recognized activities are associated with each occupant using the proposed Thermal Vision Sensor Feature Fusion $\left(T V S_{F F}\right)$ method per frame.

\subsection{Computer vision-based occupant detection and} tracking

This Section describes the inner details of the proposed framework to detect the presence of multi-occupants in real-time, and track them throughout the duration of $T V S_{-} F_{\text {seq }}$ by following them from frame-by-frame. Fig. 2 illustrates the overall $u M o D T$ framework with underlying several components, namely TVS sensor as an Input device, TVS-F Preprocessing, Occupant Tracking, and TVS-F Feature Extraction. These components are connected in series whereas the information flow between subcomponents is discussed further in the following subsections. 


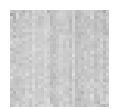

(a)

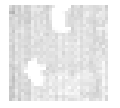

(i)

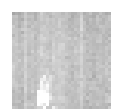

(b)

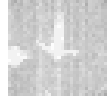

(j)

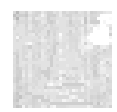

(c)

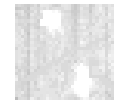

(k)

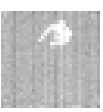

(d)

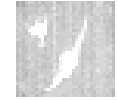

(1)

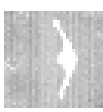

(e)

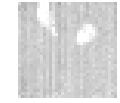

(m)

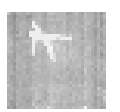

(f)

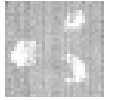

(n)

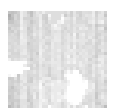

(g)

(h)

Fig. 3: (a) Empty smart living room. Single occupant activities shown as (b) Sitting (c) Standing (d) Walking (e) Stretching (f) Fall Down. Multi-occupant activities shown as (g) Two persons Sitting (h) One person Sitting while other Standing (i) One person Sitting while other Walking (j) One person Standing while other Fall Down (k) Both persons Standing (l) One person Standing while other Stretching (m) Both persons Walking (n) All are Walking (o) one person Walking while other one Stretching

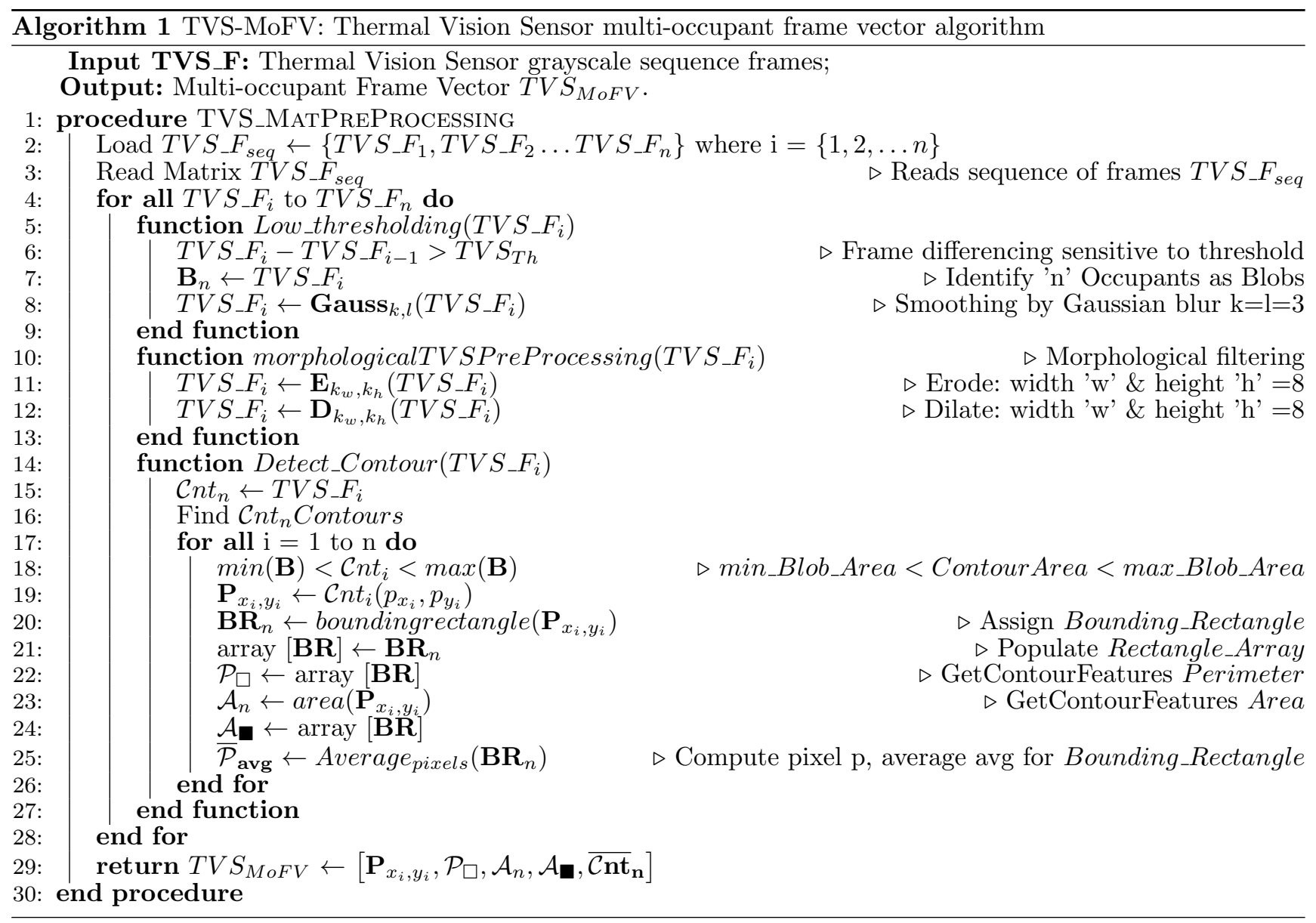

\subsubsection{Input frames}

In this study, we propose to mount the Heimann HTPA TVS [39] in the ceiling of the smart-home's living room and kitchen at the height of $3 \mathrm{~m}$. The monitored space is a quadrilateral area with dimensions $4 \times 3.5 \mathrm{~m}$. This setting provides a clear field of view and collects an aerial view of the multi-occupants as seen in Fig. 3.
It also overcomes the challenges related to occupantto-occupant and, occupant-to-scene occlusion, whilst avoiding camera motion and is operative even in complete darkness. The TVS ensures a high degree of user's privacy by capturing low-resolution grayscale $T V S_{-} F_{\text {seq }}$ with the dimensions of $32 \mathrm{~h} \times 31 \mathrm{v} \times 1$. Each of the 992 pixels correspond to an area within the smart living room and kitchen represented by each pixel value 
ranging between 0 and 255. This range sets a correspondence of every pixel with an average temperature characteristic to that area. The $T V S_{-} F_{\text {seq }}$ is managed by using RESTful HTTP services, which are processed by the server.

\subsubsection{Multi-occupant Feature Vector (TVS-MoFV)}

The frames represent the presence of heat sources within the $T V S_{-} F_{\text {seq }}$. The characteristics of identified heat sources are calculated by using the proposed Thermal Vision Sensor Multi-occupant Feature Vector (TVS-MoFV) algorithm. It gathers multi-occupant feature vectors in $T V S \_F_{\text {seq }}$ frame-by-frame. The series of tasks performed by TVS-MoFV are described in Algorithm 1] which are summarized as follows:-

- Converts the JSON $32 \times 31$ matrices into the sequence of frames $T V S_{-} F_{\text {seq }}$.

- Segments the $T V S \_F_{n}$ frames in order to detect foreground (multi-occupant) and background (static smart living room or kitchen) per frame.

- Applies the Low_thresholding TV $S_{T h}$ function with a background subtraction method sensitive to threshold 40.

- Convolves the TVS-F using Gaussian Kernel Guass $_{k, l}$ for smoothing and reducing noise with the kernel $\mathrm{k}=\mathrm{l}=3$.

- Performs morphological filtering and binarization on $T V S_{-} F_{n}$ to reduce the thermal noise using operations such as Erode $\mathbf{E}_{k_{w}, k_{h}}$ and Dilate $\mathbf{D}_{k_{w}, k_{h}}$.

- Determines the presence of multi-occupant using connected pixels termed as the contours $\mathcal{C} n t_{n}$ represented by blobs in the sequence of binary frames $T V S \_F_{n}$.

- Assigns and encapsulates each identified $\mathcal{C} n t_{i}$, within the ROI, represented by Bounding Rectangles i.e. $\mathbf{B R}_{n}$.

- Estimates the centroid $\mathbf{P}_{x_{i}, y_{i}}$ for the identified $\mathcal{C} n t_{i}$ surrounded by $\mathbf{B R}_{n}$, which acts as a pivot for further tracking.

- Computes an array of the morphological feature vector for every $T V S_{-} F_{i}$ frame, which includes Perimeter $\mathcal{P}_{\square}$, Area $\mathcal{A}_{\square}$, and Contour Pixel Average $\overline{\mathcal{P}}_{\text {avg }}$ for every $\mathbf{B R}_{n}$ in the $T V S_{-} F_{i}$.

The learned frame vector $T V S_{M o F V}$ from every TVS$\mathrm{F}$ comprises of the morphological states of the detected occupant. These states represent the occupant's thermal area, a center of contour, a perimeter of the bounding box, and the area enclosed within the bounding box encapsulating the occupant. These multiple features become the basis for $T V S_{M o A R}$ with the support of the proposed method $T V S_{F F}$ required for the data associ- ation before recognizing and associating individual activities.

\subsubsection{Multi-occupant Detection and Tracking (TVS-MoDT)}

Algorithm 2 describes the TVS Multi-occupant Detection and Tracking (TVS-MoDT) method to identify, predict, plot, visualize, and maintain the occupant's tracks within $T V S_{-} F_{s e q}$. Some of the key features for this algorithm are summarized as below:-

- The $T V S \_F_{\text {seq }}$ is read as input simultaneously as in the case of Algorithm 1

- The detected contours $\mathcal{C} n t_{i}$ through Algorithm 1 are iterated within $T V S \_F_{\text {seq }}$ for computing the vector point $\mathcal{V}_{p}$ responsible for tracking and maintaining the history of the tracks as shown in Line 4-11.

- For every detection $\mathbf{D}$ for $\mathcal{C} n t_{i}$ the tracks $\mathcal{T}_{i}$ are initialized as shown in Line 15.

- We used two classical efficient methods, Hungarian method, and KF to handle the occupant's data association and smoother motion refinement with position prediction of the multi-occupant respectively.

- The optimal assignment $\overrightarrow{\mathcal{A}}$ and cost $\mathcal{C}$ computation task for tracks $\mathcal{T}_{i}^{\text {assign }}$ is performed using the Hungarian method.

- We employed KF to generate multi-occupant motion trajectories i.e. estimation and position prediction for the blob representing each of the individual occupants as mentioned in the Line 32.

- The UpdateKalman prediction function predicts the position of the occupant based on the history from previous TVS-F whereas the update function rectifies the state of the multi-occupant from the current TVS-F (Lines 39-45).

- Every multi-occupant being tracked is assigned Tracking ID $\left(\mathcal{T}_{i d}\right)$ representing tracklets. The morphological features such as position, size and other statistical measurements are also calculated for blob.

$-\mathcal{T}_{i d}$ is dynamically assigned (or reassigned) to blobs with rapidly varying sizes. The array with tracking identifiers represents each occupant's motion model and state history.

\subsection{CNN-based activity classification}

The CNN has been utilized for real-time multi-occupant AR from the $T V S_{-} F_{s e q}$. It is computationally built on five major mathematical functions such as Convolution, Batch Normalization, Rectified Linear Unit 


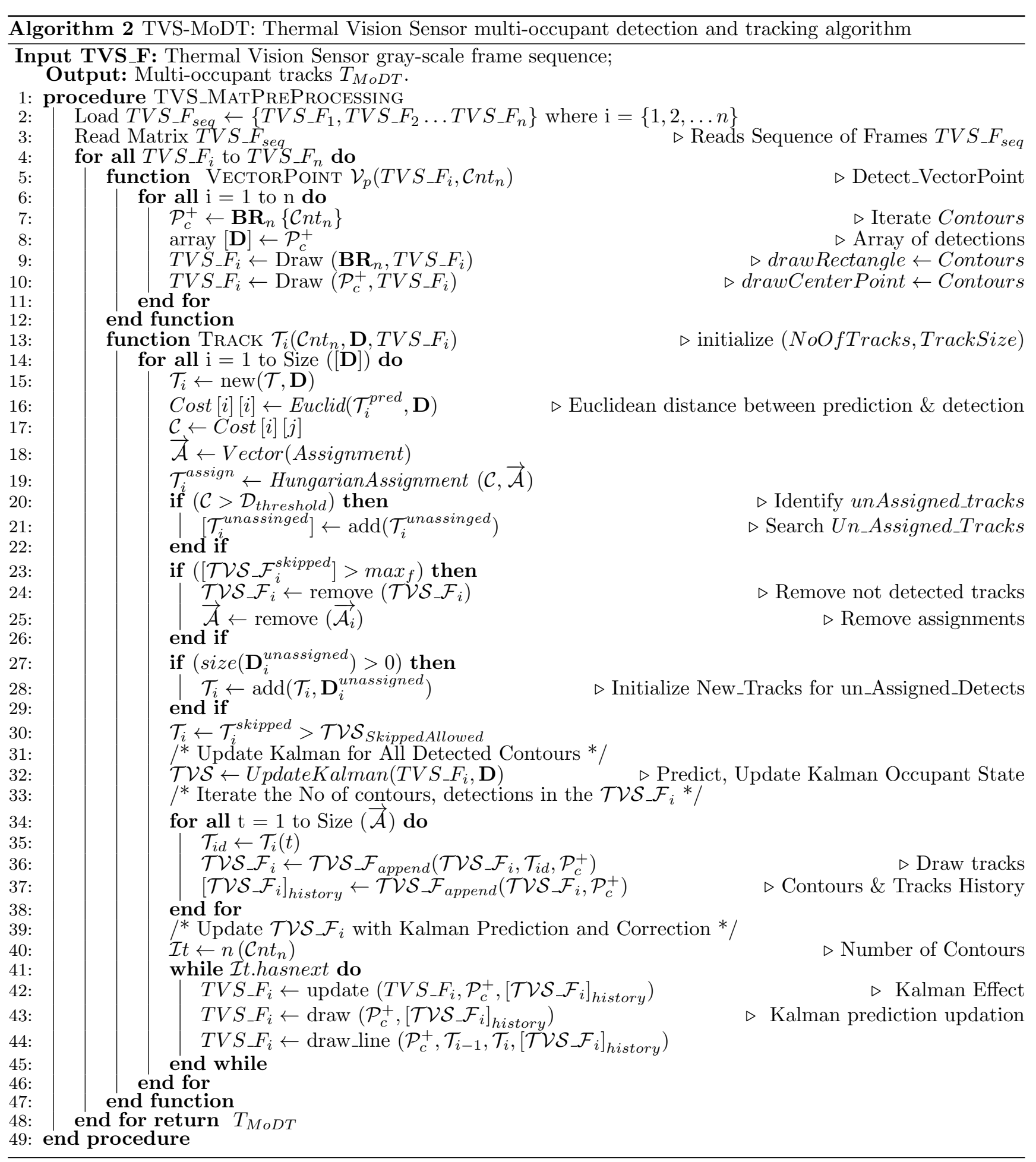

(ReLU), Pooling, and Soft-max. These functions are applied in a hierarchical residual block within an architecture, which provides fully connected layers for processing $T V S_{-} F_{\text {seq }}$ to get multi-occupant activity classification output per frame. These are briefly discussed in the following subsections.

\subsubsection{Input layer}

An input layer for the CNN architecture reads the grayscale $T V S_{-} F_{s e q}$ of the fixed dimensionality, requires $T V S \_F_{\text {Train }}$ to train the model while producing an output TVS_F $F_{\text {labelled }}$, representing "n" activities 
performed by the multi-occupants.

$T V S \_F_{\text {labelled }} \leftarrow\left\{T V S \_F_{\text {seq }}, T V S \_F_{\text {Train }}, a c t_{n}\right\}_{C N N}$

\subsubsection{Convolutional layer}

The Convolutional Layer is responsible for extracting the pixel-wise features from the input TVS-F. To learn the TVS-F features, the kernel weights are adjusted automatically through back-propagation training. The convolution is obtained by taking dot product $(\bullet)$ between sub-part of the TVS-F and the convolutional kernel $K$. In response, a feature map $f_{c}$ is computed by sliding the convolutional kernel over the TVS-F spatially. The output $x_{i}^{l, j}$ for the $l^{t h}$ convolutional layer having the $j^{t h}$ feature map on the $i^{\text {th }}$ unit can be presented mathematically as:

$x_{i}^{l, j}=\sigma\left(b_{j}+\sum_{a=1}^{m} w_{a}^{j} x_{i+a-1}^{l-1, j}\right)$

where $\sigma$ is a non-linear mapping, it uses hyperbolic tangent function, $\tanh (\cdot)$ [4].

\subsubsection{Batch normalization layer}

The input channel $x$ across the mini-batch is normalized $\hat{x}_{i}$ by the introduction of a batch normalization layer 42. Normalized activation is computed using minibatch mean $\mu_{B}$, standard deviation $\sigma_{B}^{2}$ for input channel $x$, and $\epsilon$ to provide the numeric stability for minibatch variances, described as:

$\hat{x_{i}}=\frac{x_{i}-\mu_{B}}{\sqrt{\sigma_{B}^{2}+\epsilon}}$

It increases the performance of CNN training and reduces sensitivity of the neural nets.

\subsubsection{ReLU layer}

Rectified Linear Unit (ReLU), a nonlinear activation function responsible for introducing a point-wise nonlinearity to the CNN by resolving the vanishing gradient problem [43. ReLU layer processes an element-wise activation function over each individual input $\mathrm{x}$, wherever the value is less than zero, is set to zero and it also linearly conveys the input for positive inputs described by Eq. 4

$f_{\tau}=\operatorname{ReLU}\left(x_{i}\right)=\left\{\begin{array}{l}x_{i}, x_{i} \geq 0 \\ 0, x_{i}<0\end{array}\right.$

A rectified feature map $f_{\tau}$ is obtained as an outcome.

\subsubsection{Max-pooling layer}

The max-pooling layer produces compact feature space by taking the sub-samples of $f_{\tau}$ thus reducing the spatial dimensionality and sensitivity of the output. The pooling operation derives maximum value from the set of nearby inputs as mentioned in equation 2, which can also be represented mathematically as 31]:

$f_{i}^{l, j}=\max _{r \in R}\left(x_{i \times T+r}^{l, j}\right)$

where $\mathrm{R}$ represents pooling size and $\mathrm{T}$ as a pooling stride. The soft-max classifier is placed at the final layer for HAR. The TVS-F features obtained from the stacked convolutional and pooling are represented as:

$f^{l}=\left[f_{1}, f_{2}, f_{3} \ldots f_{K}\right]$

where $\mathrm{K}$ represents the number of units learned from the last pooling layer, which acts as a feature map for the soft-max classifier.

\subsubsection{Training process}

The CNN is trained in a supervised learning fashion by selecting the parameters using Gradient-based optimization method. For faster convergence, the stochastic gradient descent method is applied [44. The training process involves a series of steps such as propagation and weight update. The gradients are computed in the propagation step by using standard forward [41] and back-propagation algorithms [45, by minimizing the objective function, which is given mathematically as:

$x_{i}^{l}=\sum_{j} w_{j, i}^{l-1} \sigma\left(x_{i}^{l-1}\right)+b_{i}^{l-1}$

where $x_{i}^{l}$ represents the output feature and $w$ is the weight vector. The output feature map is passed to every subsequent layer till it reaches the output layer, which is formulated as:

$\frac{\partial L}{\partial y_{i, j}^{l-1}}=\sum_{a=0}^{m-1} \frac{\partial L}{\partial x_{i-a}^{l}} \frac{\partial x_{i-a}^{l}}{\partial y_{i, j}^{l-1}}=\sum_{a=0}^{m-1} \frac{\partial L}{\partial x_{i-a}^{l}} w_{a, b}$

It applies chain-rule for computing the propagation error and the whole process remains cyclic until the CNN reaches a satisfactory validation state or attains the stopping criterion. 
Table 1: List of 16 activities recorded for data collection

\begin{tabular}{|c|c|c|c|}
\hline Activity ID & Activity Type & Activity Name & No. of Occupants \\
\hline$A c t_{1}$ & Single & FallDown & 1 \\
\hline$A c t_{2}, A c t_{3}$ & Single, Multi & Sitting & 1,2 \\
\hline$A_{c t}$ & Multi & SittingStanding & 2 \\
\hline Act $_{5}$ & Multi & SittingWalking & 2 \\
\hline$A c t_{6}, A_{c t}$ & Single, Multi & Standing & 1,2 \\
\hline$A c t_{7}$ & Multi & StandingFallDown & 2 \\
\hline Act $_{9}$ & Multi & StandingStretching & 2 \\
\hline Act $_{10}$ & Multi & StandingWalking & 2 \\
\hline Act $_{11}$ & Single & Stretching & 1 \\
\hline$A_{c t}, A_{12} c t_{15}, A c t_{16}$ & Single, Mutli & Walking & $1,2,3$ \\
\hline Act $_{13}$ & Multi & WalkingFallDown & 2 \\
\hline Act $_{14}$ & Multi & WalkingStretching & 2 \\
\hline
\end{tabular}

\subsubsection{Classification}

The soft-max regression function in the final layer of the neural network leads to the multi-occupant HAR using TVS-based Activity Recognition (TVS-AR) method. It normalizes the output, which is computed by fully connected layers, and more often is a combination of a set of positive numbers with their sum equivalent to one, and value ranges between $[0 \ldots 1]$. These ranges are further transformed into classification probabilities through the Classification layer in the CNN residual block. The $i$-th probability value for soft-max function $p\left(y_{i}\right)$ [4] is computed as:

$\hat{y}_{i}=p\left(y_{i}\right)=\operatorname{softmax}\left(x_{i}\right)=\frac{\exp \left(x_{i}\right)}{\sum_{k=1}^{n} \exp \left(x_{k}\right)}, i=1 \ldots N_{c}$

The cross-entropy [45] is minimized between the output probability vector $\hat{y}$ and total number of class labels 'y' as follows:

$E=-\sum_{i=1}^{N_{c}}\left(y_{i} \log \left(\hat{y}_{i}\right)+\left(1-y_{i}\right) \log \left(\hat{y}_{i}\right)\right), i=1 \ldots N_{c}$

where $y_{i}$ represents binary indicator if the class label 'c' is correctly classified from the $i^{\text {th }}$ neuron and $\hat{y}$ is the predicted probability of the $i^{t h}$ class.

\section{Experiments}

The complete real-time prototype application for our proposed $u M o D T$ framework is built for multioccupant detection, tracking and AR. To demonstrate the functionality of the $u M o D T$ framework, we first discuss the dataset and later the implementation insights.

\subsection{Dataset}

We collected 57,290 frames in a sequence from three healthy male volunteers aging $25 \pm 7$ [yrs]; height $1.55 \pm 0.7[\mathrm{~m}]$ and weight $68 \pm 8[\mathrm{~kg}]$. Each volunteer performed different ADLs individually and collectively in a smart living room over a duration of at least 3 minutes each, reported in Table 1. During the entire collection, the application was neither reparameterized nor recalibrated, which means this setting remained valid for all kind of ADLs performed during this study. Additionally, $T V S \_F_{\text {seq }}$ was annotated with LabelImg, an open source annotation tool [47. During labeling, multi-occupants were approximated by using bounding rectangles over the subsequent frames by assigning them unique identifiers referred as ground-truth $\mathbf{G}_{i}$ in the $T V S_{-} F_{s e q}$. This process followed a strict annotation protocol by qualified researchers.

The goal is to quantitatively evaluate the proposed $u M o D T$ framework and prove its accuracy and robustness. For this, we tested and compared it, also on five challenging, publicly available annotated sequences from VOT-TIR2016 challenge [48, 49]. These sequences were mostly captured with the help of static FLIR and thermal cameras.

\subsection{Implementation details}

The proposed $u M o D T$ framework, comprising of TVSMoFV (Algorithm 1), TVS-MoDT (Algorithm 2) and $T V S-A R$ method, was implemented. The former algorithms utilize the Java-based standard libraries OpenCV (an open-source API) 50, while the latter method requires MATLAB interfaces (machine learning toolbox API). The $u M o D T$ framework was imple- 
Table 2: List of benchmark dataset sequences and their details

\begin{tabular}{ccccccc}
\hline ID & Dataset & Sensor & Resolution & Frames & Object & Threshold \\
\hline 1 & ETHZ-CLA [51] & FLIR TAU320 & $324 \times 256$ & 659 & Human & 115 \\
2 & Soccer [4, 48] & $3 \times$ AXIS Q-1922 & $1920 \times 480$ & 3,000 & Human & 120 \\
3 & Crouching [48] & FLIR A655SC & $640 \times 480$ & 625 & Human & 125 \\
4 & Depthwise Crossing [48] & FLIR A655SC & $640 \times 480$ & 858 & Human & 135 \\
5 & Crowd [4] & FLIR Photon 320 & $640 \times 512$ & 78 & Human & 110 \\
6 & $T V S_{-} F_{\text {seq }}$ & Heimann & $32 \times 31$ & 57,290 & Human & 155
\end{tabular}

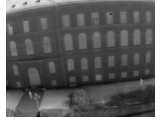

(a)

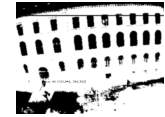

(b)

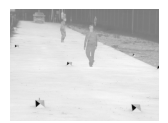

(e)

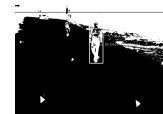

(f)

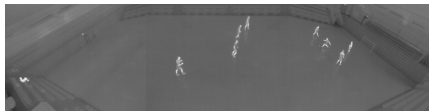

(c)

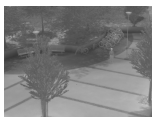

$(\mathrm{g})$

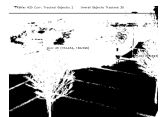

(h)

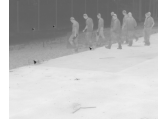

(i)

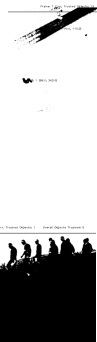

(j)

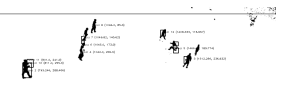

(d)

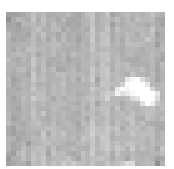

(k)

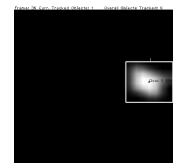

(l)

Fig. 4: Examples of raw Input (I) frames and processed Output (O) frames using proposed framework. (a) \& (b) ETHZ-CLA (I\&O) (c) \& (d) Soccer (I\&O) (e) \& (f) Crouching (I\&O) (g) \& (h) Depthwise Crossing $(\mathrm{I} \& \mathrm{O})(\mathrm{i}) \&(\mathrm{j})$ Crowd $(\mathrm{I} \& \mathrm{O})(\mathrm{k}) \&(\mathrm{l})$ TVS-F $(\mathrm{I} \& \mathrm{O})$

mented and evaluated using the PC system equipped with AMD A10-5800K APU with Radeon(tm) HD Graphics (4 CPUs 3.8GHz), 16GB RAM, and NVIDIA GeForce GTX 750 GPU 4GB.

Proposed algorithms, TVS-MoFV for feature extraction and TVS-MoDT for multi-occupant detection and tracking were tested. Both of them used stored $T V S_{-} F_{\text {seq }}$, which was retrieved from the intermediate repository as JSON object arrays, by a pull-based webservice. In TVS-MoFV, TVS-F vector was obtained by varying binary threshold values and finding the best

Table 3: Processing time for benchmarks and $T V S_{-} F_{\text {seq }}$ with TVS-MoDT and TVS-AR algorithms

\begin{tabular}{ccc}
\hline Algorithm & Dataset & Duration(s) \\
\hline \multirow{4}{*}{ TVS-MoDT } & ETHZ-CLA & $3.91 \times 10^{-6}$ \\
& Soccer & $2.99 \times 10^{-6}$ \\
& Crouching & $6.35 \times 10^{-6}$ \\
& Depthwise Crossing & $2.93 \times 10^{-6}$ \\
& Crowd & $2.93 \times 10^{-6}$ \\
TVS-AR & $T V S_{-} F_{\text {seq }}$ & $4.88 \times 10^{-6}$ \\
& $T V S_{-} F_{\text {seq }}(O=1)$ & $7.1 \times 10^{-2}$ \\
& $T V S_{\text {seq }}(O=2)$ & $8.3 \times 10^{-2}$ \\
& & \\
& &
\end{tabular}

value, suitable for each of VOT-TIR2016 benchmark datasets and the $T V S_{-} F_{\text {seq }}$ as mentioned in Table 2 The parametric settings also involved finding the optimal value for the contour area in order to predict the maximum number of occupants in the benchmarks and TVS_F $F_{\text {seq }}$ as shown in Fig. 4. These TVS-F feature vectors support while iterating the multi-occupant represented as Blobs predicted as bounding rectangles, implemented through the TVS-MoDT algorithm. The Euclidean distance was calculated between the detected and predicted bounding rectangles for multi-occupant tracking frame-by-frame. The processing time for each algorithm and method to process a single frame is referred to in Table 3 . The source code for $u M o D T$ framework and $T V S_{\_} F_{\text {seq }}$ is available on GitHub at [52].

To recognize multi-occupant's ADLs from $T V S_{-} F_{\text {seq }}$, a supervised CNN model was trained. For this the entire collection of $T V S_{-} F_{\text {seq }}$ was sorted into two subset groups i.e. training and test categories, each having sixteen classes. The training set is further split with random TSV-F distribution into two halves i.e. $70 \%$ for training samples $\left(T V S_{-} F_{\text {Train }}\right)$ and remaining to validate each class. We used 28,485 TVS-F samples to train CNN model and 1,920 TVS-F test samples (120 TVS-F for each of 16 classes) to evaluate the prototype $u M o D T$ framework application. 
Table 4: TVS-AR: Activity recognition for multi-occupants using Convolution Neural Networks

\begin{tabular}{|c|c|c|c|}
\hline Layer & Layer Type & Activation & Parameters (No. of units, Size, Stride) \\
\hline 1 & $T V S \_F_{s e q}$ & Image Input & $32 \times 32 \times 1$ images with zerocenter normalization \\
\hline 2 & conv1 & Convolution & $163 \times 3 \times 1$ convolutions with stride $\left[\begin{array}{ll}1 & 1\end{array}\right]$ and padding $\left[\begin{array}{llll}1 & 1 & 1 & 1\end{array}\right]$ \\
\hline 3 & batchnorm1 & Batch Normalization & Batch normalization with 16 channels \\
\hline 4 & relu1 & ReLU & ReLU \\
\hline 5 & maxpool1 & Max Pooling & 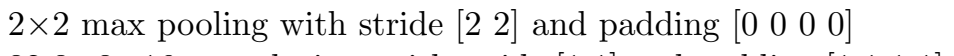 \\
\hline 6 & conv2 & Convolution & $323 \times 3 \times 16$ convolutions with stride [ $\left[\begin{array}{ll}1 & 1\end{array}\right]$ and padding $\left[\begin{array}{lll}1 & 1 & 1\end{array}\right]$ \\
\hline 7 & batchnorm2 & Batch Normalization & Batch normalization with 32 channels \\
\hline 8 & relu2 & ReLU & ReLU \\
\hline 9 & maxpool2 & Max Pooling & 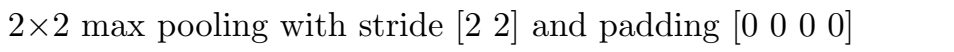 \\
\hline 10 & conv3 & Convolution & $323 \times 3 \times 32$ convolutions with stride $\left[\begin{array}{ll}1 & 1\end{array}\right]$ and padding $\left[\begin{array}{lll}1 & 1 & 1\end{array}\right]$ \\
\hline 11 & batchnorm3 & Batch Normalization & Batch normalization with 32 channels \\
\hline 12 & relu3 & ReLU & ReLU \\
\hline 13 & maxpool3 & Max Pooling & 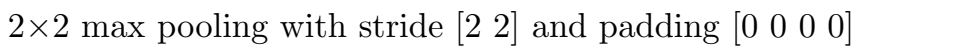 \\
\hline 14 & $\operatorname{conv} 4$ & Convolution & $643 \times 3 \times 32$ convolutions with stride $\left[\begin{array}{ll}1 & 1\end{array}\right]$ and padding $\left[\begin{array}{lll}1 & 1 & 1\end{array}\right]$ \\
\hline 15 & batchnorm4 & Batch Normalization & Batch normalization with 64 channels \\
\hline 16 & relu4 & ReLU & ReLU \\
\hline 17 & fc & Fully Connected & 16 fully connected layers \\
\hline 18 & soft-max & soft-max & Bayesian binary classifier \\
\hline 19 & classoutput & Classification Output & crossentropyex with FallDown and 15 other classes \\
\hline
\end{tabular}

The nineteen-layer, CNN architecture is designed based on the findings from the systematic comparison and benchmarking to achieve an affordable classification time and computation cost [53. The implemented $\mathrm{CNN}$ architecture comprises of two units i.e. feature extractor and a non-linear classifier [29. The former unit encapsulates fifteen layers (Layer2... Layer16) whereas the latter unit i.e. non-linear classifier is built on all fully connected layers along with the soft-max classifier. During the model training process, the CNN hyperparameters were set with the help of input functions, by adjusting the learning rate effectively to 0.01 , every 10 epochs using Stochastic Gradient Descent with Momentum (SGDM) algorithm with the maximum 20 number of epochs size [45. For every iteration, a minibatch of size 16 (64) was applied for which the details are mentioned in Table 4. The output of the last ReLU (relu4) at Layer 16, is given to fully connected layer Layer 17, which uses the features and processes it for class prediction based on the TVS $S_{\text {Train }}$. The classification layer i.e. Layer 18 uses the soft-max activation function, which squashes the output probability vector between sixteen multi-occupant activities and returns the binary indicator to them.

\section{Results and discussion}

In literature there exist several performance measures to deal with single-target and multi-target tracking, however, none of them proved to be a defacto standard. In our experiments, we adopted some of the effective multi-occupant detection and tracking evaluation strategies to: a) detect and track the multi-occupants and b) classify multi-occupant activities in $T V S_{-} F_{\text {seq }}$. For this, we investigated frame properties in the sequences to identify the influence of different parameters such as variable thresholds and overlap measures on the overall performance. Moreover, conformity of evaluation measures to any other application and sequence have been proven by the $u M o D T$ framework on VOTTIR2016 sequences other than TVS_F $F_{\text {seq }}$.

\subsection{Multi-occupant detection and tracking evaluation}

Objectively quantitative assessment of multi-occupant detection and tracking is not a straight forward task. Most of the evaluation techniques require a groundtruth $G_{i}$, which serves as a reference to measure the performance quantitatively. We adopted such evaluation methods, which rely on frame based spatial overlap between $\mathrm{G}_{\mathrm{i}}$ and bounding rectangles $\mathrm{BR}_{\mathrm{n}}[54$.

\subsubsection{Evaluation metrics}

The object detection in benchmark sequences and multi-occupant detection in $T V S_{-} F_{\text {seq }}$ uses standard Pascal, Intersection over Union (IoU) criterion, a natural bounding box evaluation measure for comparing 


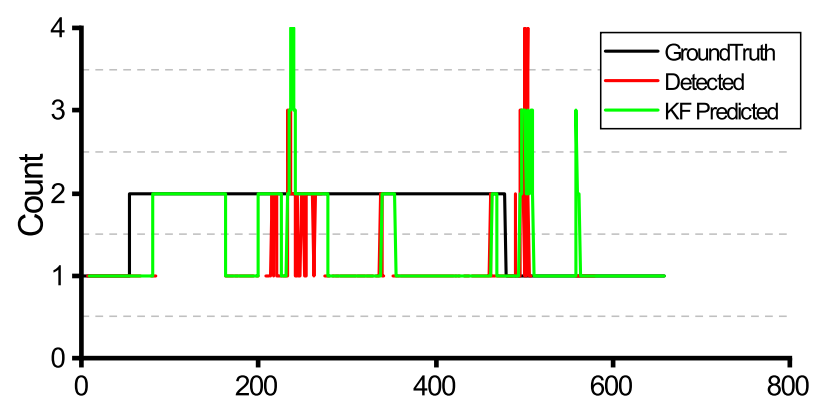

(a) ETHZ-CLA Frame Sequence

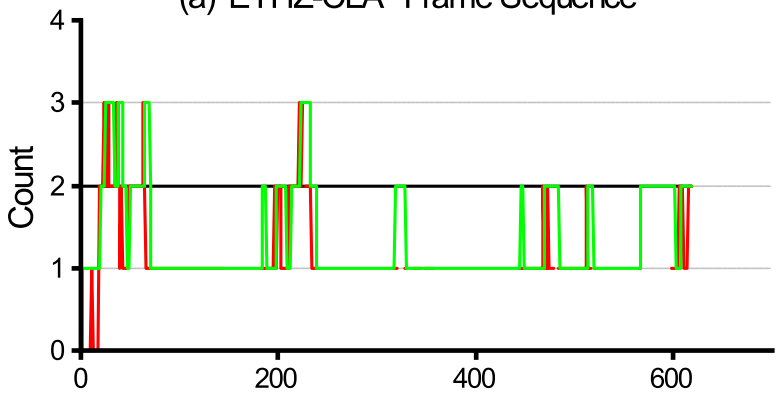

(c) Crouching Frame Sequence

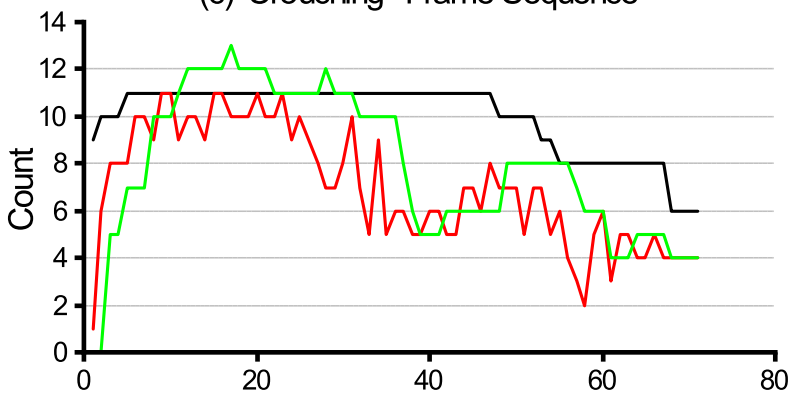

(e) Crowd Frame Sequence

Fig. 5: Quantitative evaluations shown in (a) ETHZ-CLA (b) Soccer (c) Crouching (d) Depthwise Crossing (e) Crowd (f) TVS-F

spatial overlap and localization accuracy [48]:

$I o U\left(B R_{\mathrm{n}}, G_{\mathrm{i}}\right)=\frac{B R_{\mathrm{n}} \cap G_{\mathrm{i}}}{B R_{\mathrm{n}} \cup G_{\mathrm{i}}}$

\subsubsection{Performance evaluation and analysis}

We take the advantage of the Counting algorithm to estimate number of occupants against $\mathrm{G}_{\mathrm{i}}$ frame-wise in each of the sequence [55. In our experiments, we considered count detection as true positive (TP) for IoU greater than 0.5 otherwise as false positive (FP). For Io $U<0.5$, however, we also considered rotated $\mathrm{BR}_{\mathrm{n}}$ locations for each object obtained from KF in the frame to see if updated object state has any spatial overlap relation with ground-truth. Fig. 5(a-f) present results for $\mathrm{G}_{\mathrm{i}}$, detected, and $\mathrm{KF}$ predicted $\mathrm{BR}_{\mathrm{n}}$ frame-wise in each sequence. The best counting success rate is achieved by using improved frame pre-processing algorithms TVS-

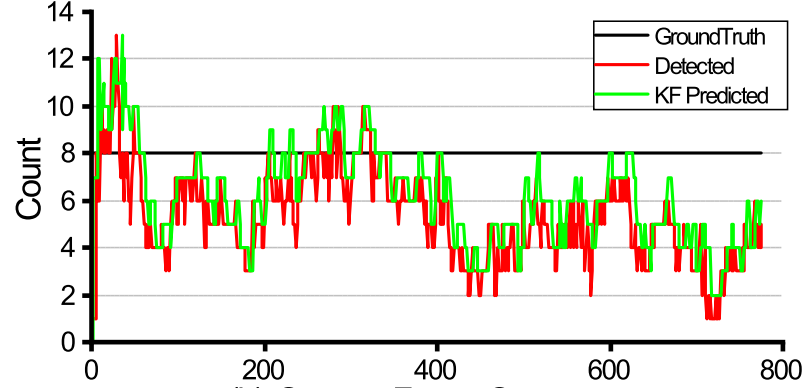

(b) Soccer Frame Sequence

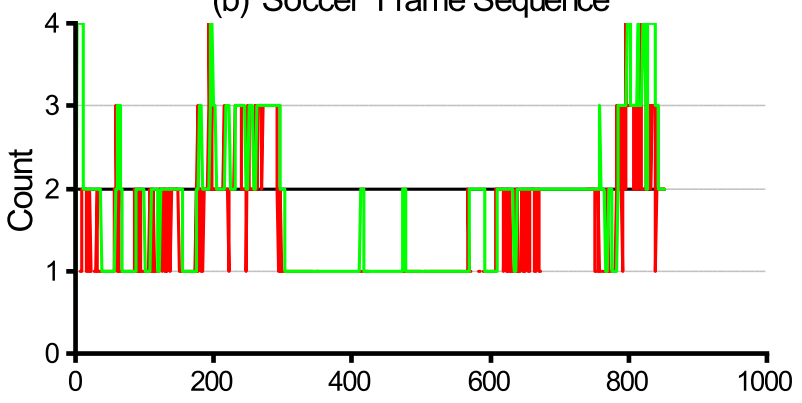

(d) DepthWiseCrossing Frame Sequence

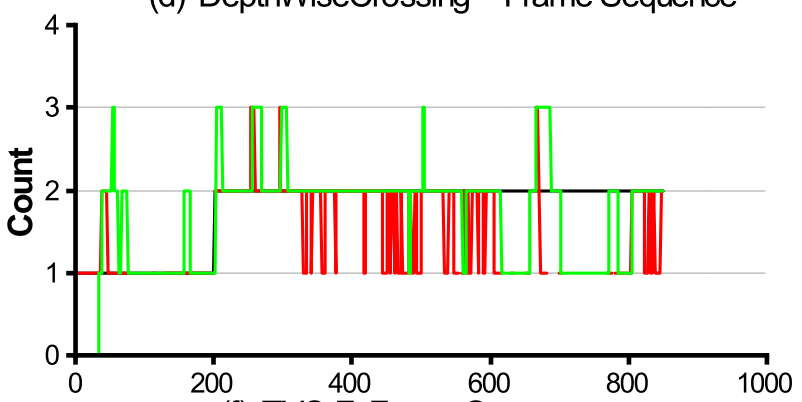

(f) TVS-F Frame Sequence 
Table 5: Evaluation comparison of the uMoDT framework for benchmark sequences and $T V S_{\_} F_{\text {seq }}$

\begin{tabular}{|c|c|c|c|c|c|c|c|c|}
\hline & Name & $\mathbf{F P} \downarrow$ & $\mathbf{F N} \downarrow$ & MOTA $\uparrow$ & IDS $\downarrow$ & Precision $\uparrow$ & Recall $\uparrow$ & MSE $\downarrow$ \\
\hline \multirow{6}{*}{ 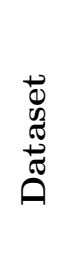 } & ETHZ-CLA & 441 & 414 & 5.58 & 210 & 0.61 & 0.44 & 1.04 \\
\hline & Soccer & 311 & 1540 & 74.42 & 246 & 0.94 & 0.39 & 5.19 \\
\hline & Crouching & 163 & 428 & 57.17 & 243 & 0.80 & 0.29 & 1.08 \\
\hline & Depthwise & 456 & 408 & 53.03 & 180 & 0.72 & 0.38 & 0.96 \\
\hline & Crowd & 110 & 211 & 57.40 & 110 & 0.81 & 0.41 & 12.27 \\
\hline & $T V S \_F_{s e q}$ & 52 & 469 & 64.26 & 72 & 0.87 & 0.42 & 0.84 \\
\hline
\end{tabular}

Table 6: Evaluation comparison for the uMoDT framework against other techniques

\begin{tabular}{|c|c|c|c|c|c|}
\hline & Name & $\mathbf{F P} \downarrow$ & $\mathbf{F N} \downarrow$ & $\overline{\text { MOTA } \uparrow}$ & IDS $\downarrow$ \\
\hline \multirow{7}{*}{ 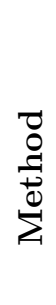 } & Bochinski et al. 57 & 5702 & 70278 & 57.1 & 2167 \\
\hline & Wan et al. 58 & 10604 & 56182 & 62.6 & 1389 \\
\hline & Bewley et al. [59] & 7318 & 32615 & 33.4 & 1001 \\
\hline & Murray et al. 60] & 3130 & 76202 & 27.4 & 786 \\
\hline & Chen et al. 61] & 9253 & 85431 & 47.6 & 792 \\
\hline & Gade et al. [55] & $9.8 \%$ & $18.8 \%$ & 70.36 & 219 \\
\hline & $u M o D T\left(T V S \_F_{\text {seq }}\right)$ & 52 & 469 & 64.26 & 72 \\
\hline
\end{tabular}

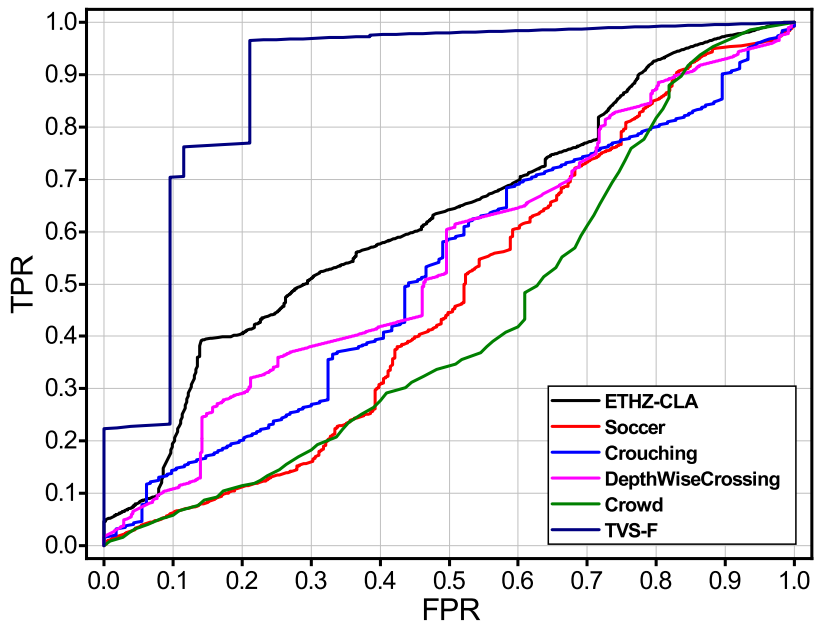

Fig. 6: ROC curves for benchmark sequences and TVS_F $F_{\text {seq }}$

sources of error, MOTA is defined as:

$\operatorname{MOT} A=1-\frac{\sum_{t}\left(F N_{t}+F P_{t}+I D S_{t}\right)}{\sum_{t} G_{t}}$

We report quantitative evaluations and comparative analysis through the experiments over a set of test sequences for frame-based detection and tracking in Tables 5 and 6 respectively. It is evident that the $u M o D T$ framework demonstrated better performance in terms of MOTA for benchmark sequences and $T V S_{-} F_{\text {seq }}$. It outperformed other techniques on all sequences especially for Soccer sequence and TV $S_{-} F_{\text {seq }}$ with MOTA scores of $74.42 \%$ and $64.26 \%$ respectively. Additionally, the Mean Squared Error (MSE) between the localization of predicted $\mathrm{BR}_{\mathrm{n}}$ and $\mathrm{G}_{\mathrm{i}}$ was also computed as:

$M S E=\frac{1}{n} \sum_{i=1}^{n}\left(B R_{\mathrm{n}}-G_{\mathrm{i}}\right)^{2}$

The error rates showed lowest MSE value of 0.84 , which was achieved for $T V S_{-} F_{\text {seq }}$ and a highest MSE value of 12.27 for Crowd sequence. The tabulated results, however, showed a higher number of IDS $_{t}$, an increased MSE, and a decreased MOTA, which appeared to be from occlusions and deforming blobs.

The performance of $u M o D T$ is also compared by constructing ROC curves for accumulated true detection rates and false positive rates using $G_{i}$ and predicted $\mathrm{BR}_{\mathrm{n}}$ with $I o U>0.5$ as shown in Fig. 6. The ROC curve produced by $T V S_{-} F_{\text {seq }}$ has shown a larger area under the curve than other sequences. This suggests and validates the robustness of the proposed algorithm for occupant detection. $T V S_{-} F_{\text {seq }}$ has lessor FPR, which is due to minimal occlusion as compared to other sequences especially in Crowd sequence, which has maximum occlusion. Fig. 7 shows the resulting precisionrecall curves based on overlap metric. Such a quantitative analysis proves as how successfully the $\mathrm{BR}_{\mathrm{n}}$ are predicted for $\mathrm{G}_{\mathrm{i}}$ in the benchmark sequences and $T V S_{-} F_{\text {seq }}$. The $u M o D T$ framework achieved a highest 


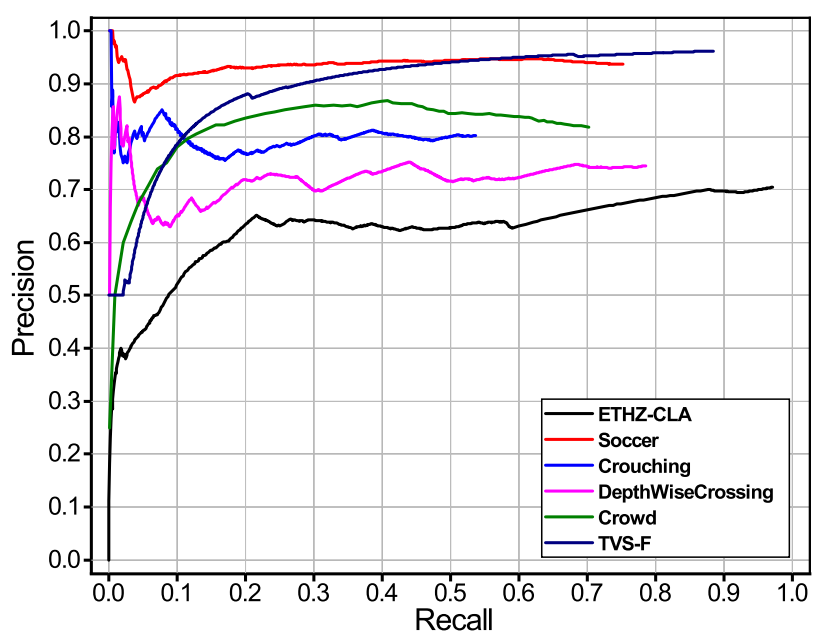

Fig. 7: Precision-recall curves for benchmark sequences and $T V S_{-} F_{\text {seq }}$

area under the curves with an average $97.16 \%$ precision rate for $T V S_{-} F_{\text {seq }}$ and the lowest one with around $72.04 \%$ for $E T H Z-C L A$ sequence.

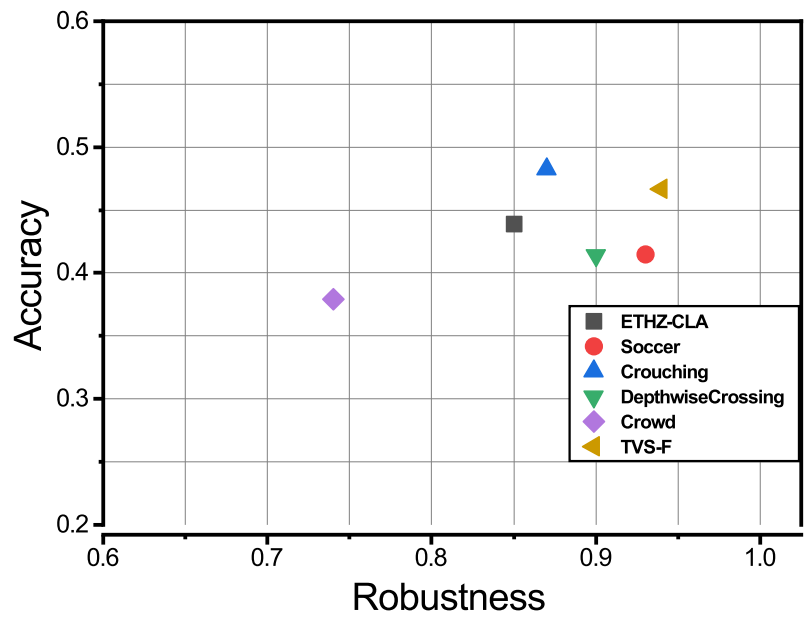

Fig. 8: Accuracy-robustness plot for the $u M o D T$ with benchmarks and $T V S_{-} F_{\text {seq }}$

\subsection{3 uMoDT robustness}

To assess the ability of the $u M o D T$ framework as how it deals with the tracking failure, we further quantify it for robustness measure correlated with accuracy. Robustness refers to the $u M O D T$ failures whenever the overlap $I o U$ measure becomes equal to zero. To measure the average overlap areas and complete failures, these measures are intuitively computed for benchmark sequences with $I o U$ threshold value equal to zero. We also assumed each occupant in a frame as a separate entity, represented by an independent motion trajectory to evaluate tracking performance 62. The resulting robustness, however, in some cases does not have an upper bound so it was interpreted as a reliability, defined by $e^{-S\left(F_{0} / N\right)}$ for visualizing purpose 63, 64. Here $\mathrm{N}$ denotes number of frames for an individual sequence, $\mathrm{S}$ represents the number of frames since the last failure, and $F_{0}$ is a failure rate, which is set as $I o U$ equal to zero. We executed the $u M o D T$ framework separately for each sequence to record their average scores, failure rate and unsupervised re-initialization for multioccupants.

Fig. 8 demonstrates the effectiveness of the $u M o D T$ framework, which proved to be most robust on TVS$F$ sequence (positioned most right) but it was surpassed by Crouching sequence, which appeared to be more accurate (positioned higher). The observed high robustness for $T V S_{-} F_{\text {seq }}$ is because of no occlusion, static distinguishable background and quality of multioccupant estimates using KF. On the other hand, high average accuracy for Crouching sequence is observed, which is due to frequent re-initialization as occupant's appearance is challenging which matches with background. The $u M o D T$ framework performed differently between the benchmark sequences depending on their frame properties, however, it achieved an overall best performance except for the Crowd sequence (positioned lowered). At a closer look, we see that in terms of accuracy it is challenging as occupants are not well distinguishable from background and also frequent $u M o D T$ failures occur due to occlusions. It still, however, has achieved satisfactory robustness.

\subsection{Multi-occupant activity recognition}

In the following subsection, to show the generality of the TVS-AR method, we describe and evaluate the proposed CNN-based model using the TV $S_{-} F_{\text {seq }}$ for AR. We present the classification results to prove the performance and suitability of the presented approach using low-resolution $T V S_{-} F_{\text {seq }}$ in terms of accuracy 65. We used frame-based approach for recognizing 16 different activities showing the efficacy of a model by demonstrating it for a high HAR accuracy score of approximately $90.99 \%$.

\subsubsection{Activity recognition evaluation metrics}

The performance metric that is most widely used to evaluate a classifier in the context of multiclass classifi- 
Table 7: Average accuracy confusion matrix for multioccupant HAR

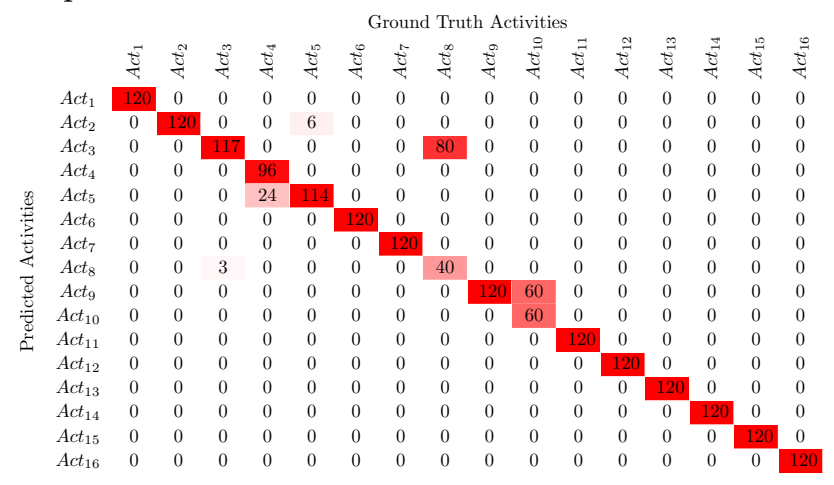

cation is overall accuracy [41]. The recognition accuracy is linear to the number of training frames. The training frames were used to fit in the parameters such as weights, validation set to fine tune the parameters and CNN architecture. The performance of the customized CNN was evaluated on validation split as a test data to validate the generalization and prediction power of the classifier. Additionally, the other most common performance evaluation metrics such as precision, recall, Fmeasure also provided an essential information required to assess the classification model [43].

\subsubsection{Performance evaluation of activities}

For each experiment, we followed the data splits and cross-validation evaluation technique for TVS_F seq. We divided $T V S_{-} F_{\text {seq }}$ into three splits: training split TVS_F $F_{\text {Train }}$ to train CNN model, validation split to tune the hyper-parameters such as learning rate, epoch size on unseen data, and finally test split to evaluate the classification performance. An average accuracy of $97.34 \%$ was achieved with a learning rate of 0.01 for 28,485 TVS_F $F_{\text {seq. }}$. A drop in accuracy, however, was observed with a decrease in the learning rate. The test split contained 1,920 TVS-F for validating 16 activities as mentioned in the confusion matrix illustrated through Table 7. It is observed that the TVS-AR method accurately classified most of singleoccupant and multi-occupant activities. Nevertheless, some confusion has been observed for multi-occupant activities such as StandingWalking (Act 10$)$ and StandingStretching (Act 9 ) have been confused due to similar motion patterns for Walking and Stretching. This is due to the activity Stretching, which involves extension of arms and returning to their original position, again sharing motion patterns to the activity Standing in a TVS_F $F_{\text {seq }}$. Similarly, static multi-occupant activities SittingSitting $\left(\right.$ Act $\left._{3}\right)$ and StandingStanding $\left(\right.$ Act $\left._{8}\right)$

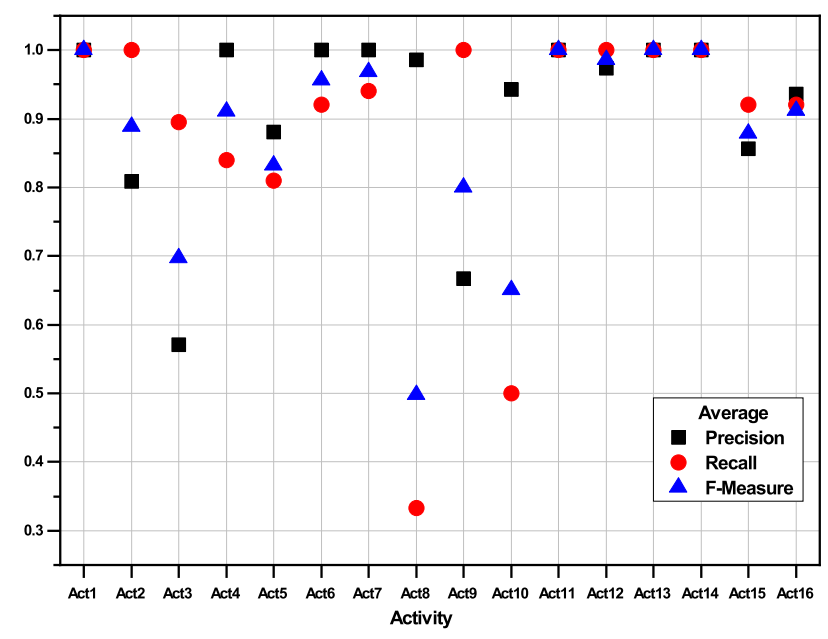

Fig. 9: Classification accuracy using CNN for test TVS$\mathrm{F}$

share similar occupant appearances in the TVS_F seq. For these, the activities Standing and Sitting were confused due to similar heat maps in the frames. Furthermore, Fig. 9 shows the evaluation metrics in terms of Precision, Recall and F-Measure. By visualizing these, it can be concluded that multi-occupant activity i.e. $\left(\right.$ Act $\left._{8}\right)$ with both occupants Standing and $\left(\right.$ Act $\left._{10}\right)$ with one occupant Standing and other one Walking has shown the lowest performance for the test split of TVS_F $F_{\text {seq }}$.

\section{Conclusions}

In this work, we proposed and demonstrated an unobtrusive Multi-occupant Detection and Tracking $(u M o D T)$ framework for HAR based on low resolution TVS. In this study, by using a binarization technique with Gaussian filter for smoothing, a morphological improvement with inversion and dilation process, an individual occupant in the form of the blob was detected over a sequence of frames. This blob was further tracked by using a $\mathrm{KF}$ with location improvement and evaluated with Intersection over Union (IoU). The above methods achieved detection and tracking accuracy of $88.46 \%$ for Thermal Vision Sensor frame sequence $\left(T V S_{-} F_{\text {seq }}\right)$. Additionally, a CNN-based multioccupant HAR method was evaluated, achieving a validation accuracy of $97.34 \%$ and an accuracy of $90.99 \%$ for classification tasks. This experimentation demonstrates improvements in occupant detection and, activity association using TVS. The experimental evaluation using state-of-the-art benchmark datasets also revealed the robustness and effectiveness of the proposed frame- 
work. Further improvements may be achieved by introducing multiple TVS(s) for HAR. These settings may include movable TVS to recognize ADLs for more complex scenarios at different indoor locations.

Acknowledgements This research was supported by an Institute for Information \& Communications Technology Promotion (IITP) grant funded by the Korean government (MSIT) (No. 2017-0-00655). This work was supported by the MSIT (Ministry of Science and ICT), Korea, under the ITRC (Information Technology Research Center) support program (IITP-2017-0-01629) supervised by the IITP (Institute for Information \& communications Technology Promotion) and NRF-2016K1A3A7A03951968. This work was supported by the REMIND project, which has received funding from the European Union's Horizon 2020 research and innovation programme under the Marie Skłodowska-Curie grant agreement No 734355 .

\section{References}

1. Benmansour A, Bouchachia A, Feham M (2016) Multioccupant activity recognition in pervasive smart home environments. ACM Computing Surveys (CSUR) 48(3):34

2. Chen L, Hoey J, Nugent CD, Cook DJ, Yu Z (2012) Sensor-based activity recognition. IEEE Transactions on Systems, Man, and Cybernetics, Part C (Applications and Reviews) 42(6):790-808

3. Singla G, Cook DJ, Schmitter-Edgecombe M (2010) Recognizing independent and joint activities among multiple residents in smart environments. Journal of ambient intelligence and humanized computing 1(1):57-63

4. Gade R, Moeslund TB (2018) Constrained multitarget tracking for team sports activities. IPSJ Transactions on Computer Vision and Applications 10(1):2

5. Synnott J, Rafferty J, Nugent CD (2016) Detection of workplace sedentary behavior using thermal sensors. In: Engineering in Medicine and Biology Society (EMBC), 2016 IEEE 38th Annual International Conference of the, IEEE, pp 5413-5416

6. Fiaz M, Mahmood A, Jung SK (2018) Tracking noisy targets: A review of recent object tracking approaches. arXiv preprint arXiv:180203098

7. Tran SN, Zhang Q, Karunanithi M (2018) On multi-resident activity recognition in ambient smart-homes. arXiv preprint arXiv:180606611

8. Gade R, Moeslund TB, Nielsen SZ, Skov-Petersen H, Andersen HJ, Basselbjerg K, Dam HT, Jensen OB, Jørgensen A, Lahrmann H, et al (2016) Thermal imaging systems for real-time applications in smart cities. International Journal of Computer Applications in Technology 53(4):291-308
9. Li X, Hu W, Shen C, Zhang Z, Dick A, Hengel AVD (2013) A survey of appearance models in visual object tracking. ACM transactions on Intelligent Systems and Technology (TIST) 4(4):58

10. Shen J, Liang Z, Liu J, Sun H, Shao L, Tao D (2018) Multiobject tracking by submodular optimization. IEEE Transactions on Cybernetics

11. Wang J, Chen Y, Hu L, Peng X, Philip SY (2018) Stratified transfer learning for cross-domain activity recognition. In: 2018 IEEE International Conference on Pervasive Computing and Communications (PerCom), IEEE, pp 1-10

12. Wang L, Gu T, Tao X, Chen H, Lu J (2011) Recognizing multi-user activities using wearable sensors in a smart home. Pervasive and Mobile Computing $7(3): 287-298$

13. Rafsanjani HN, Ahn CR, Alahmad M (2015) A review of approaches for sensing, understanding, and improving occupancy-related energy-use behaviors in commercial buildings. Energies 8(10):10,99611,029

14. Hevesi P, Wille S, Pirkl G, Wehn N, Lukowicz P (2014) Monitoring household activities and user location with a cheap, unobtrusive thermal sensor array. In: Proceedings of the 2014 ACM international joint conference on pervasive and ubiquitous computing, ACM, pp 141-145

15. Sengar SS, Mukhopadhyay S (2017) Moving object detection based on frame difference and w4. Signal, Image and Video Processing 11(7):1357-1364

16. Mandellos NA, Keramitsoglou I, Kiranoudis CT (2011) A background subtraction algorithm for detecting and tracking vehicles. Expert Systems with Applications 38(3):1619-1631

17. Xing J, Ai H, Lao S (2009) Multi-object tracking through occlusions by local tracklets filtering and global tracklets association with detection responses. In: 2009 IEEE Conference on Computer Vision and Pattern Recognition, IEEE, pp 1200 1207

18. Parekh HS, Thakore DG, Jaliya UK (2014) A survey on object detection and tracking methods. International Journal of Innovative Research in Computer and Communication Engineering 2(2):2970 2979

19. Luo W, Xing J, Zhang X, Zhao X, Kim TK (2014) Multiple object tracking: A literature review. arXiv preprint arXiv:14097618

20. Cai Z, Gu Z, Yu ZL, Liu H, Zhang K (2016) A real-time visual object tracking system based on kalman filter and mb-lbp feature matching. Multimedia Tools and Applications 75(4):2393-2409 
21. Yilmaz A, Javed O, Shah M (2006) Object tracking: A survey. Acm computing surveys (CSUR) 38(4):13

22. Luo X, Guan Q, Tan H, Gao L, Wang Z, Luo X (2017) Simultaneous indoor tracking and activity recognition using pyroelectric infrared sensors. Sensors $17(8): 1738$

23. $\mathrm{Hu}$ WC, Chen $\mathrm{CH}$, Chen TY, Huang DY, Wu ZC (2015) Moving object detection and tracking from video captured by moving camera. Journal of Visual Communication and Image Representation 30:164-180

24. Hou L, Wan W, Hwang JN, Muhammad R, Yang M, Han K (2017) Human tracking over camera networks: a review. EURASIP Journal on Advances in Signal Processing 2017(1):43

25. Zhang B, Li Z, Perina A, Del Bue A, Murino V, Liu J (2016) Adaptive local movement modeling for robust object tracking. IEEE Transactions on Circuits and Systems for Video Technology 27(7):1515-1526

26. Choi W, Savarese S (2012) A unified framework for multi-target tracking and collective activity recognition. In: European Conference on Computer Vision, Springer, pp 215-230

27. Shen J, Yu D, Deng L, Dong X (2017) Fast online tracking with detection refinement. IEEE Transactions on Intelligent Transportation Systems

28. Zebin T, Scully PJ, Ozanyan KB (2016) Human activity recognition with inertial sensors using a deep learning approach. In: 2016 IEEE SENSORS, IEEE, pp 1-3

29. Krizhevsky A, Sutskever I, Hinton GE (2012) Imagenet classification with deep convolutional neural networks. In: Advances in neural information processing systems, pp 1097-1105

30. Dhillon JK, Kushwaha AKS, et al (2017) A recent survey for human activity recoginition based on deep learning approach. In: Image Information Processing (ICIIP), 2017 Fourth International Conference on, IEEE, pp 1-6

31. Dobhal T, Shitole V, Thomas G, Navada G (2015) Human activity recognition using binary motion image and deep learning. Procedia computer science 58:178-185

32. Ji S, Xu W, Yang M, Yu K (2013) 3d convolutional neural networks for human action recognition. IEEE transactions on pattern analysis and machine intelligence 35(1):221-231

33. Ray KS, Chakraborty S (2017) An efficient approach for object detection and tracking of objects in a video with variable background. arXiv preprint arXiv:170602672
34. Leira FS, Johansen TA, Fossen TI (2015) Automatic detection, classification and tracking of objects in the ocean surface from uavs using a thermal camera. In: Aerospace Conference, 2015 IEEE, IEEE, pp 1-10

35. Tiwari M, Singhai R (2017) A review of detection and tracking of object from image and video sequences. International Journal of Computational Intelligence Research 13(5):745-765

36. Wang Y, Luo X, Fu S, Hu S (2018) Context multitask visual object tracking via guided filter. Signal Processing: Image Communication

37. Dehghan A, Shah M (2018) Binary quadratic programing for online tracking of hundreds of people in extremely crowded scenes. IEEE transactions on pattern analysis and machine intelligence $40(3): 568-581$

38. Sahbani B, Adiprawita W (2016) Kalman filter and iterative-hungarian algorithm implementation for low complexity point tracking as part of fast multiple object tracking system. In: 2016 6th International Conference on System Engineering and Technology (ICSET), IEEE, pp 109-115

39. Heimanntvs. http://www.heimannsensor.com/ productsimaging.php, accessed: 2020-02-25

40. Medina-Quero Jea, Nugent C (2018) Computer vision-based gait velocity from non-obtrusive thermal vision sensors. To be Sumitted $0(0): 1-6$

41. Zeng M, Nguyen LT, Yu B, Mengshoel OJ, Zhu J, Wu P, Zhang J (2014) Convolutional neural networks for human activity recognition using mobile sensors. In: Mobile Computing, Applications and Services (MobiCASE), 2014 6th International Conference on, IEEE, pp 197-205

42. Ioffe S, Szegedy C (2015) Batch normalization: Accelerating deep network training by reducing internal covariate shift. arXiv preprint arXiv:150203167

43. Ordóñez FJ, Roggen D (2016) Deep convolutional and lstm recurrent neural networks for multimodal wearable activity recognition. Sensors 16(1):115

44. Albelwi S, Mahmood A (2017) A framework for designing the architectures of deep convolutional neural networks. Entropy 19(6):242

45. Gao Z (2018) Object-based image classification and retrieval with deep feature representations. Doctor of Philosophy Thesis, School of Computing and Information Technology, University of Wollongong

46. Teow MY (2017) Understanding convolutional neural networks using a minimal model for handwritten digit recognition. In: Automatic Control and Intelligent Systems (I2CACIS), 2017 IEEE 2nd International Conference on, IEEE, pp 167-172 
47. Tzutalin Labelimg: Image annotation tool. https:// github.com/tzutalin/labelImg, accessed: 2020-0225

48. Kristan M, Matas J, Leonardis A, Vojir T, Pflugfelder R, Fernandez G, Nebehay G, Porikli F, Cehovin L (2016) A novel performance evaluation methodology for single-target trackers. IEEE Transactions on Pattern Analysis and Machine Intelligence 38(11):2137-2155, DOI 10.1109/TPAMI. 2016.2516982

49. Vot2016 benchmark. http://www.votchallenge. net/vot2016/, accessed: 2020-02-25

50. Bradski G (2000) The OpenCV Library. Dr Dobb's Journal of Software Tools

51. Portmann J, Lynen S, Chli M, Siegwart R (2014) People detection and tracking from aerial thermal views. In: Robotics and Automation (ICRA), 2014 IEEE International Conference on, IEEE, pp 17941800

52. $u M o D T$ framework source code. https://github. com/masifrazzaq/TVS-DTC/, accessed: 2020-0225

53. Mishkin D, Sergievskiy N, Matas J (2017) Systematic evaluation of convolution neural network advances on the imagenet. Computer Vision and Image Understanding 161:11-19

54. Manohar V, Soundararajan P, Raju H, Goldgof D, Kasturi R, Garofolo J (2006) Performance evaluation of object detection and tracking in video. In: Asian Conference on Computer Vision, Springer, pp 151-161

55. Gade R, Moeslund T (2014) Thermal tracking of sports players. Sensors 14(8):13,679-13,691

56. Bernardin K, Stiefelhagen R (2008) Evaluating multiple object tracking performance: the clear mot metrics. EURASIP Journal on Image and Video Processing 2008:1-10

57. Bochinski E, Eiselein V, Sikora T (2017) Highspeed tracking-by-detection without using image information. In: 2017 14th IEEE International Conference on Advanced Video and Signal Based Surveillance (AVSS), IEEE, pp 1-6

58. Wan X, Wang J, Zhou S (2018) An online and flexible multi-object tracking framework using long short-term memory. In: Proceedings of the IEEE Conference on Computer Vision and Pattern Recognition Workshops, pp 1230-1238

59. Bewley A, Ge Z, Ott L, Ramos F, Upcroft B (2016) Simple online and realtime tracking. In: 2016 IEEE International Conference on Image Processing (ICIP), IEEE, pp 3464-3468

60. Murray S (2017) Real-time multiple object tracking-a study on the importance of speed. arXiv preprint arXiv:170903572

61. Chen L, Ai H, Zhuang Z, Shang C (2018) Real-time multiple people tracking with deeply learned candidate selection and person re-identification. arXiv preprint arXiv:180904427v1

62. Wu Y, Lim J, Yang MH (2015) Object tracking benchmark. IEEE Transactions on Pattern Analysis and Machine Intelligence 37(9):1834-1848

63. Čehovin L, Kristan M, Leonardis A (2014) Is my new tracker really better than yours? In: IEEE Winter Conference on Applications of Computer Vision, IEEE, pp 540-547

64. Čehovin L, Leonardis A, Kristan M (2016) Visual object tracking performance measures revisited. IEEE Transactions on Image Processing 25:12611274

65. Wang Q, Gong D, Qi M, Shen Y, Lei Y (2018) Temporal sparse feature auto-combination deep network for video action recognition. Concurrency and Computation: Practice and Experience p e4487 